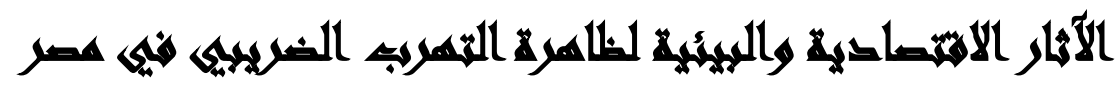

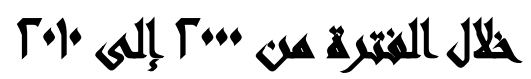

[19]

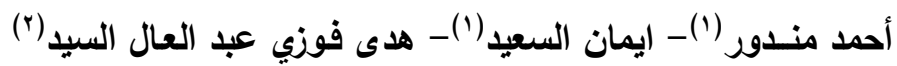

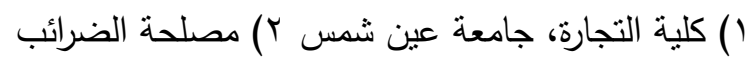

\section{المستخلص:}

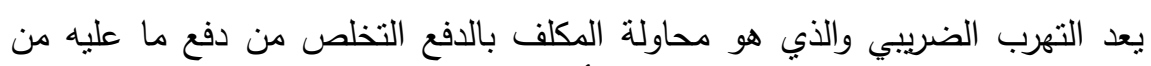

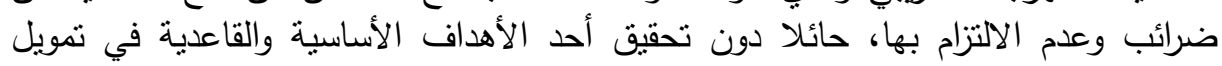

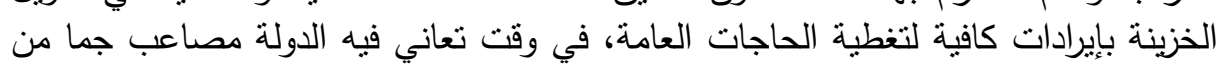

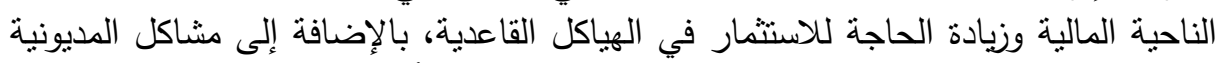

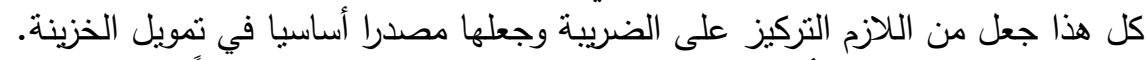

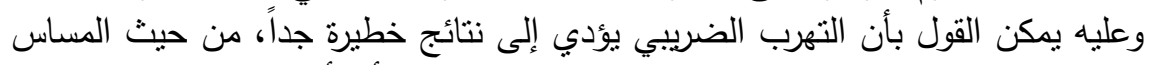

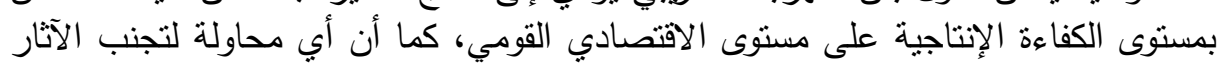

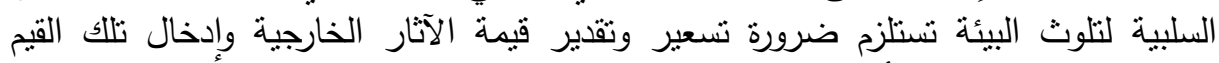

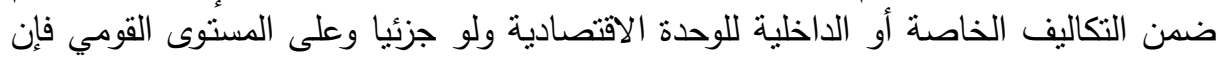

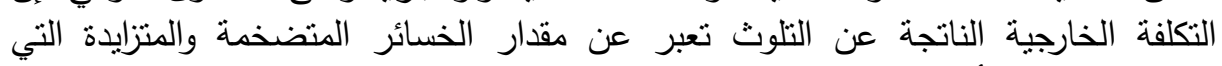

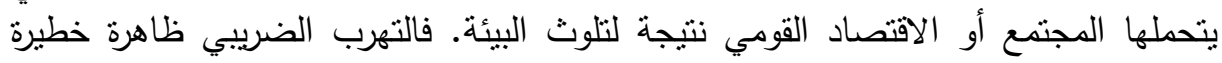

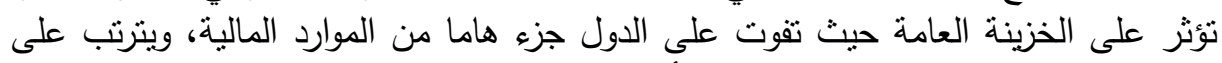

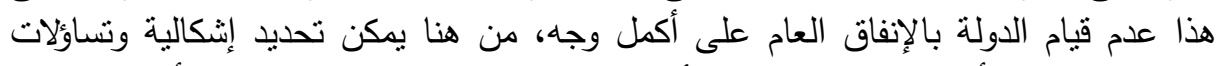

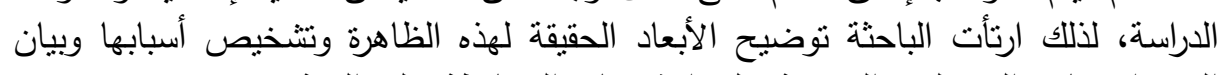

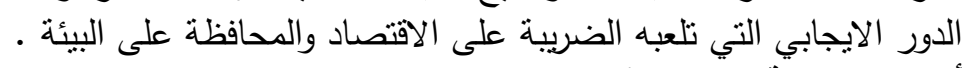

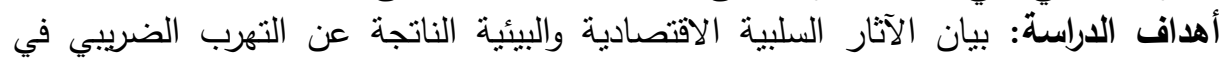

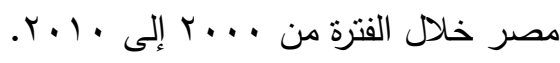

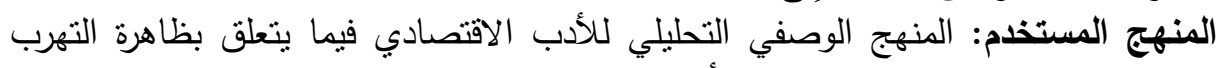

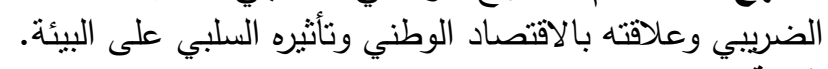

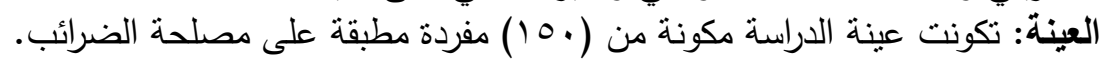

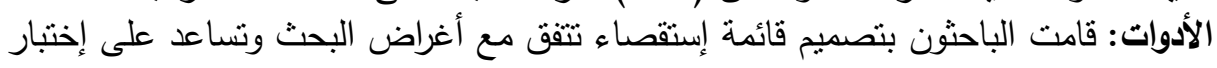

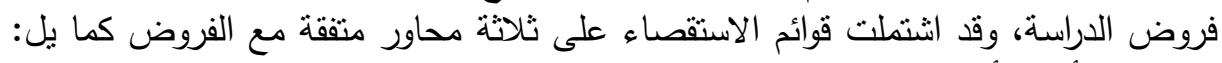

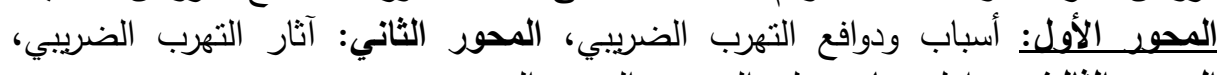

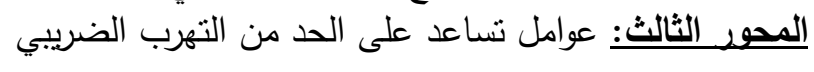

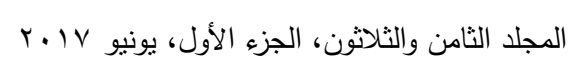


أهم نتائج الدراسة: هنالك علاقة ارتباطية ذات دلالة احصائية بين التهرب الضريبي وفاعلية

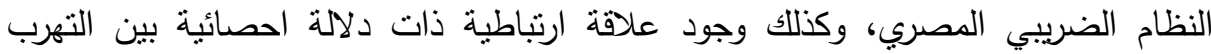

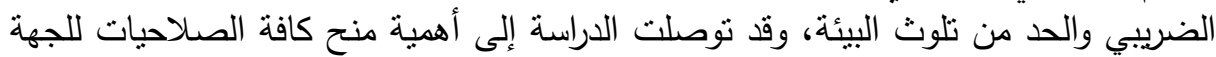

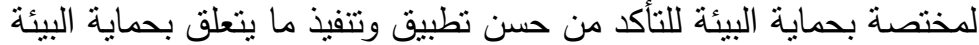

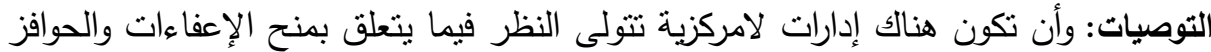

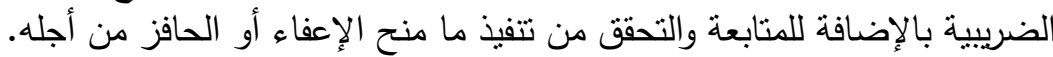

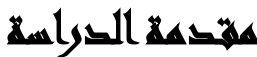

لتحقيق التتمية الثاملة كان لابد من توفير مصادر تمويلية داخلية وخارجية، ولأن مصادر التمويل الخارجي من مساعدات مالية واقتراض مشروط من الهيئات العالمية المالية غالبا ما ترتبط بانعكاسات سلبية، فقد سعت أغلب الدول إلى إيجاد مصادر تمويلية داخلية على أن تتسم بالاستقرار والدوام، وأن تكون عنصرا هاما من عناصر مالية الدولة. كل هذا أدى إلى الاهتمام بالضريبة كمورد هام لتغطية نفقات الدولة، كما نستخدمها الدولة كوسيلة فعالة لتحقيق أهدافها الاقتصادية والاجتماعية والسياسية، زيادة إلى أثرها الواضح في إرسال مبدأ العدالة الاجتماعية وتكافؤ الفرص، عن طريق إعادة نوزيع الدخل والإيرادات بين مختلف فئات المجتمع، من أجل تحسين الخدمات العامة والمستوى المعيثي

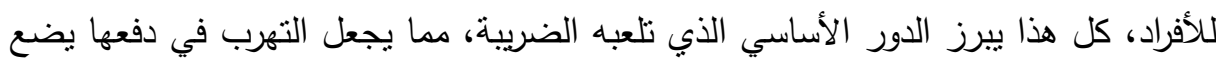

$$
\text { حدا لأهم عنصر في مالية الدولة. }
$$

وعليه، فإن ظاهرة التهرب الضريبي وكونها عالمية وجدت بتواجد الضريبة، إلا أنها

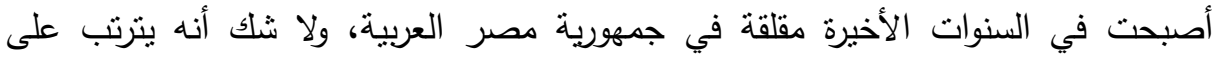
التهرب من أداء الضريبة آثارا مالية واقتصادية واجنماعية وسياسية وكنلك بيئية، يتأثر بها المتهرب كما يتأثر بها المجتمع، كما أن للتهرب آناره على أسعار السوق والتضخم والبطالة والثكل العام لميزانية الدولة.

وبالرغم من أن مشكلة البيئة بحسب تعريفها هي مشكلة اقتصادية لأن التلوث في جوهره نتيجة مباشرة للنشاط الاقتصادي، ويترتب عن طريق معالجته آنار مهمة على الأنشطة 
الاقتصادية، فإن الأسس الاقتصادية التي تقوم عليها سياسات حماية البيئة لا نزال هشة وغير

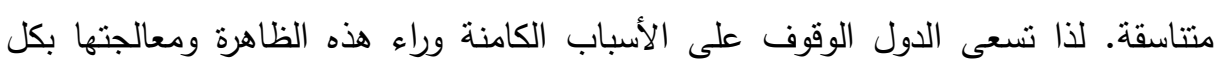

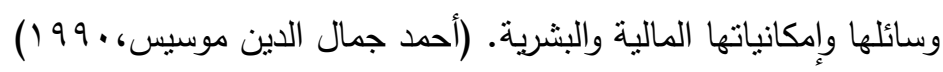
وكان هذا المنحنى في التفكير هو الباعث على إعداد هذه الدراسة التي سوف تنتاول

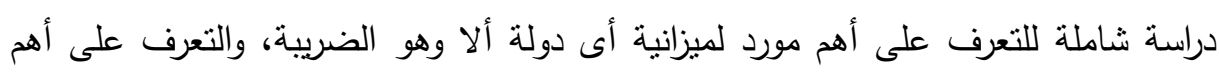

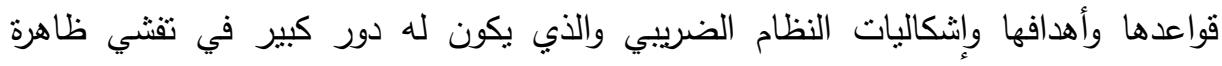

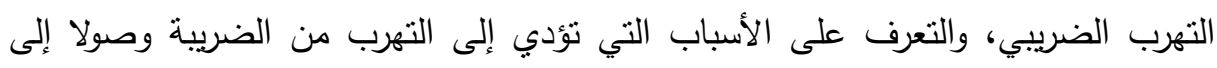

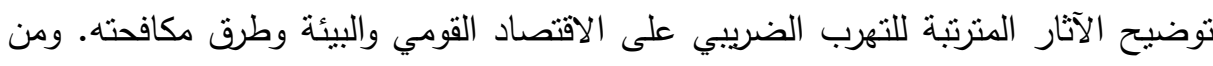

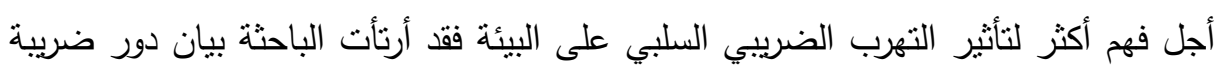

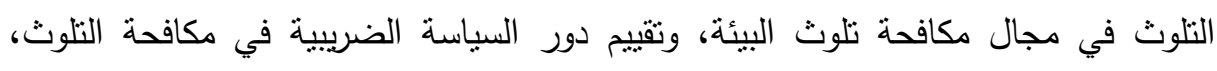

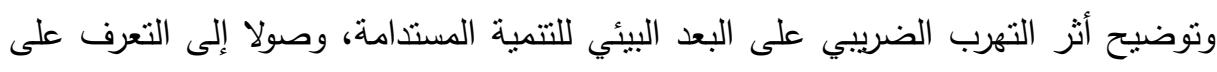
الاتجاهات الحديثة لمكافحة التهرب الضريبي.

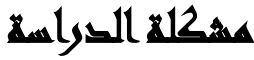

إن سعى الدولة إلى مكافحة التهرب الضريبي، يتمنل في محاولة القضاء على أسبابه، من أجل تحقيق التتمية الاقتصادية والتخلص من البطالة، وتقليص الفوارق الاجتماعية بين فئات المجتمع وغيرها من الأهداف، التي تؤدي إلي عدة تساؤلات وطرح الإثشكالية التالية:

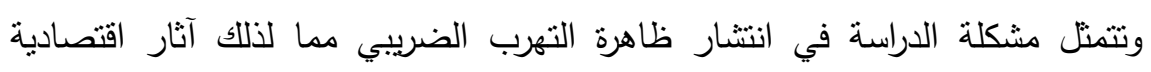
وبيئية، ومن هنا فإن مشكلة الدراسة تكمن في الآثار السلبية التي تتتج عن التهرب الضريبي

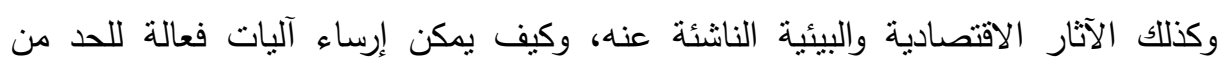
التهرب، حتى نجعل الضريبة تلعب دورها الأساسي في تمويل خزينة الدولة .

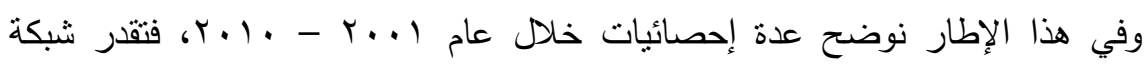

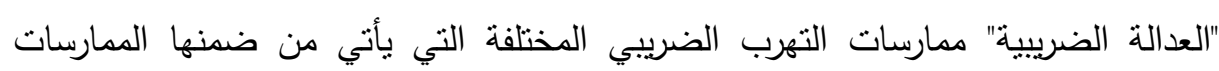

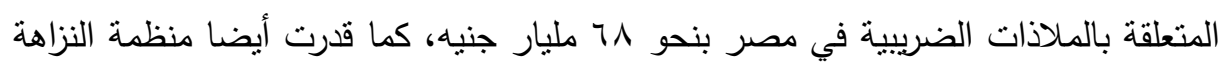
العالمية Global Financial Integrity تكلفة التلاعب بالفواتير - وهي واحدة من وسائل

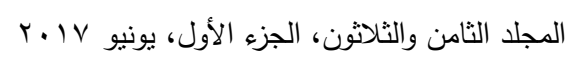




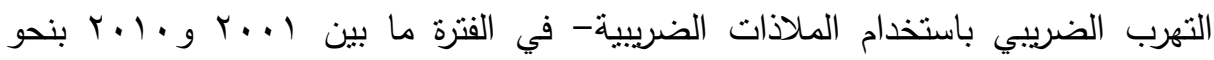

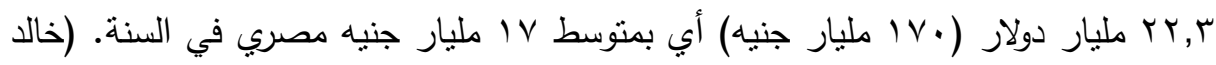

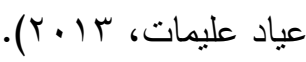
كما أثنار بيان لقطاع مكافحة التهرب الضريبي بمصلحة الضرائب المصرية حول

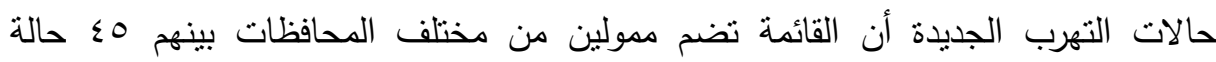

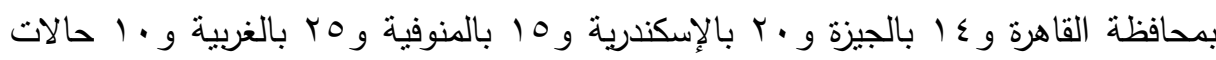

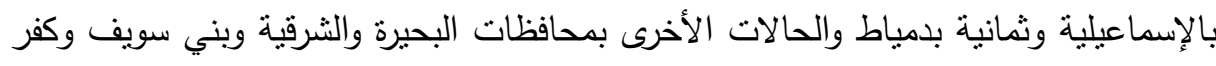
الثيخ.

وقد قام الباحثون بعمل استمارة استبيان عن التهرب الضريبي في مصر عن الفترة من

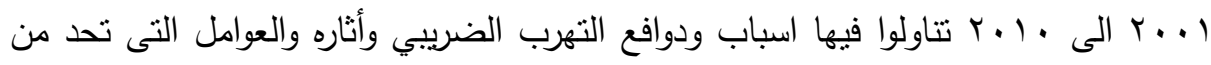
التهرب الضريبي. ويالتالي فإن الباحثون قد حددوا مشكلة الدراسة الراهنة بناءً على تساؤل رئيسي يتفرع منه عدة أسئلة فرعية على النحو التالي: ا ـ ما الآثار الاقتصادية والبيئية التي يمكن أن تتتج عن ظاهرة التهائ التهرب من الضريبة؟

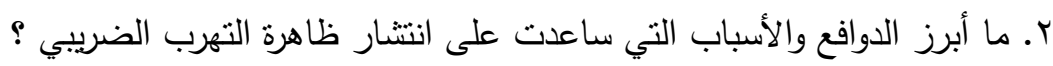

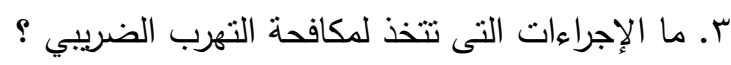

\section{أهمه التصواسم}

• تكمن أهمية البحث في الوقوف على هذه الظاهرة لتشخيص الداء ومحاولة إيجاد الدواء،

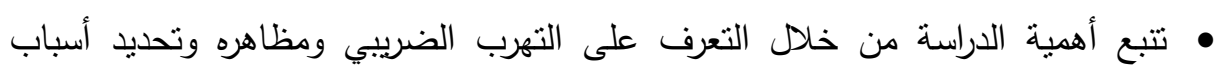

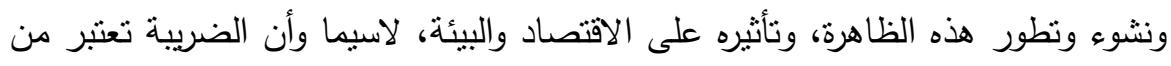

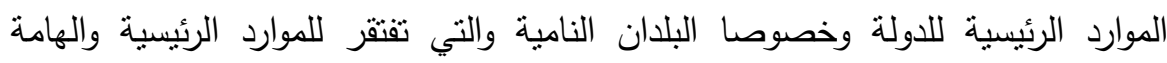

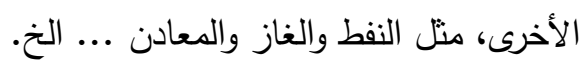


تحديد مفهوم الضريبة التي يتهرب منها المكلفين بها، والخصائص التي تميزها والوقوف هن خاصة عند المبادئ القائمة عليها الضريبة، من حيث فرضيثة فرضها وفقا لقواعد العدالة

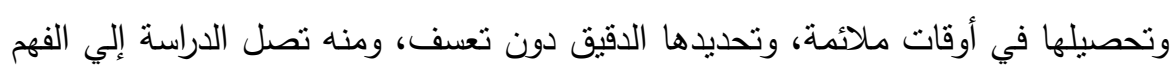
الحقيقي للاور الفعال الذي يقوم به هذا المورد الرئيسي (الضريبة) في تعزيز التتمية

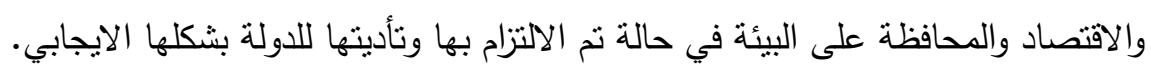

\section{أهمانهم التصراسة}

يكمن الهدف الرئيسي لهذه الدراسة في بيان الآثار السلبية الاقتصادية والبيئية الناتجة

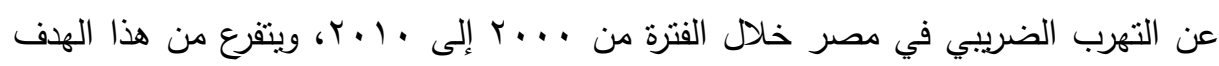
الرئيسي عدة أهداف فرعية تتمثل في الآتي:

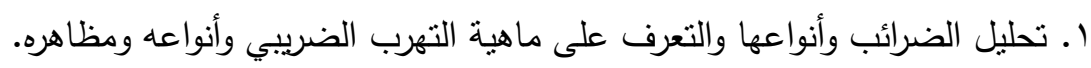
r. التعرف على أسباب انتشار مشكلة التهرب الضريبي.

r. دراسة وتحليل النتائج التي تتجم عن التهرب الضريبي خاصة الآثار الاقتصادية والبيئية. ع. دراسة وتحليل كيفية تفعيل النظام الضريبي المطبق في مصر لضمان تحقيق أعلى

$$
\text { حصيلة ضريبية. }
$$

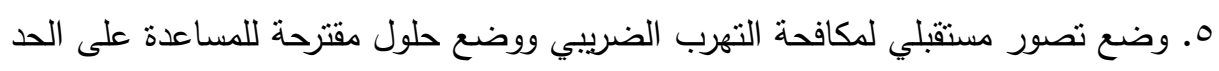
من ظاهرة التهرب الضريبي في مصر.

\section{هروض القواسمة}

من خلال الاراسة إلى عدة فرضيات:

ا ـ نوجد علاقة ذات دلالة معنوية بين التهرب الضريبي وفاعلية النظام الضريبي المصرئي الصري. r. توجد علاقة ذات دلالة معنوية بين التهرب الضريبي والحد من تلوث البيئة.

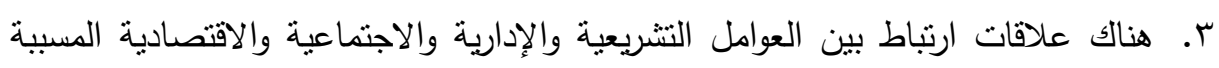
للتهرب الضريبي. ع. هناك علاقة ذات دلالة معنوية بين العوامل التي تساعد على الحد من التهرب الضريبي.

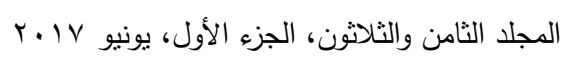




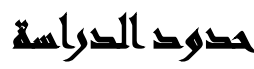

الحدود الزمنية: تغطي هذه الدراسة الآثار الناتجة عن التهرب الضريبي وتأثنيره على

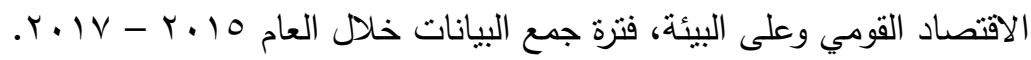
الحدود المكانية: يتحدد نطاق البحث في النزول الميداني لكل من الدائرة العامة لمصلحة الضرائب ووزارة المالية والجهاز المركزي للإحصاء عن طريق جمع البيانات وتحليلها، ويتكون

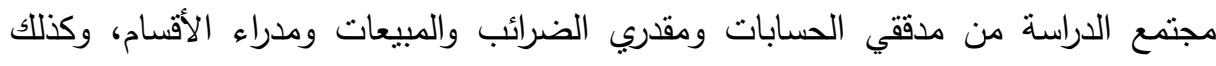
اختيار عينة عشوائية أخرى من المكلفين بالضريبة، وذللك حتى تتمكن الباحثة من تحليل ظاهرة التهرب الضريبي من منظور المكلفين بها.

\section{هنهمج التواسمة}

وللإجابة عن نساؤلات الدراسة المطروحة، كان من الضروري اتباع المنهج الوصفي

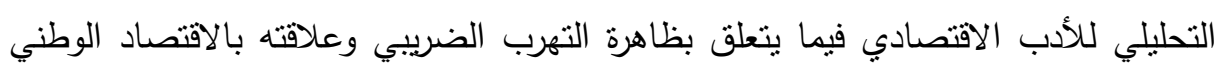

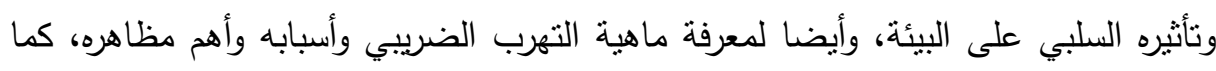

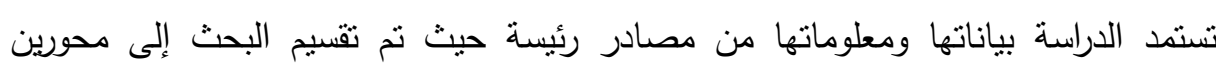
أساسيين:

أ- مصادر ثانوية جاهزة: تتمثل في المقتنيات المكتبية حيث اشتمل على إجراء مسح

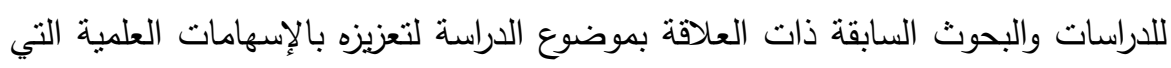
تتاولته، كان أهمها الكتب والمقالات والأبحاث العلمية والرسائل الجامعية والوثائق الرسمية. ب- مصادر أوليه: نتمثل في جمع البيانات والمعلومات مباشرة من الميدان من خلال المقابلات الثخصية مع بعض المسئولين، بواسطة استبانة تم تصميمها لغايات هذه

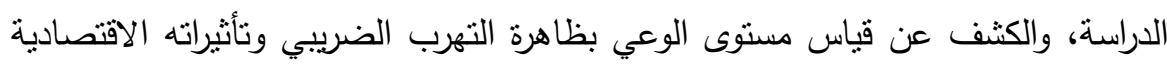
والبيئية، وذلك بالاعتماد على الدراسة الميدانية وتحليل نتائجها فضلا عن تصميم استبانه

$$
\text { تضمنت معظم الجوانب الرئيسية لمحتوى الدراسة. }
$$




\section{التوراساهي الساريخ}

على الرغم من أهية ظاهرة التهرب الضريبي للتعرف على أسبابها وأنكالها وآثارها

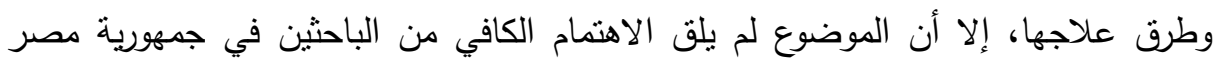

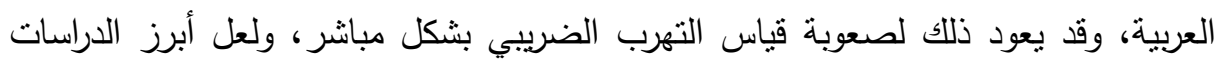
التي عالجت هذا الموضوع على المستوى الوطني والدولي هي:

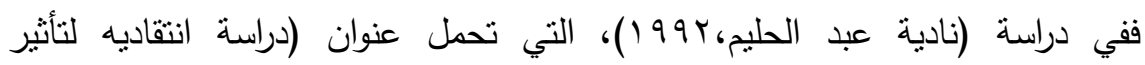
المحفزات الضريبية في القوانين المصرية على مناخ الأعمال)، وهى تهدف إلى دراسة أهمية

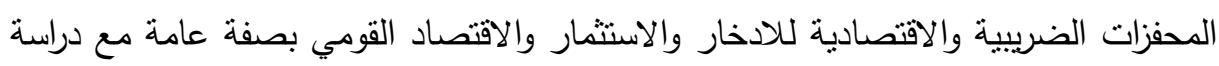
انتقاديه للمحفزات الضريبية الموجودة بالقوانين المصرية، قد تبين أن تشجيع الاستثمار يحتاج

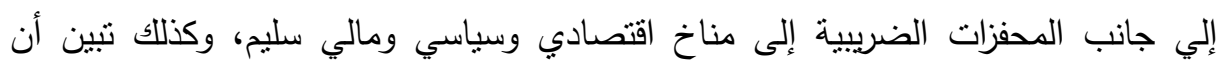
الحوافز الضريبية المطبقة في مصر قد نجحت إلي حد ما في جذب رؤوس الأموال للاستثمار في مصر إلا إنها فنثلت في توظيف تلاك الأموال في المجالات المرغوبة وهي الاستثمارات في الأصول الرأسمالية.

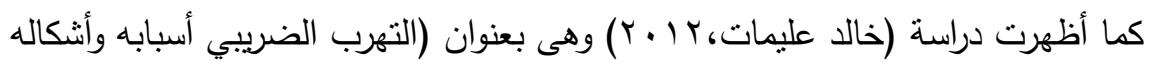
وطرق الحد منه)، والتي تهدف إلى التعرف إلى العوامل والأسباب التي تذفع المكلفين في

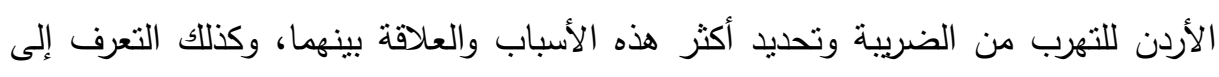
الأساليب والوسائل التي تقلل من التهرب الضريبي وبالتالي مكافحته، فقد تبين من خلال نتائج

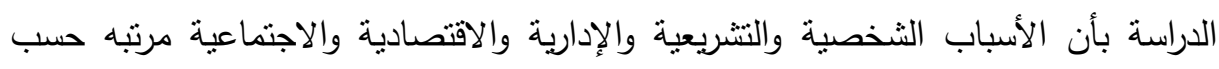

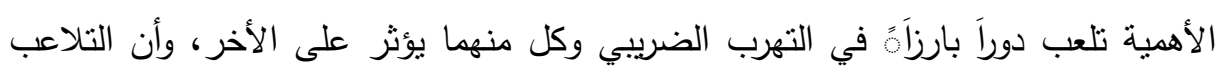

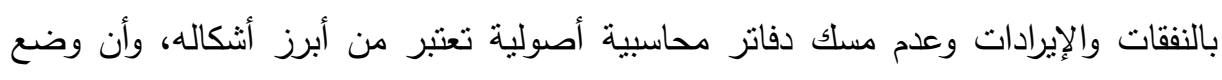
حوافز تشجيعية وتحقيق العدالة بين المكلفين تقلل من التهرب الضريبي. كما تبين من دراسة (حافظ شعيب، 1990 (1) وهى بعنوان (تأثير ضرائب المبيعات على لتئي

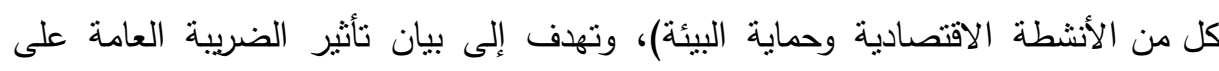

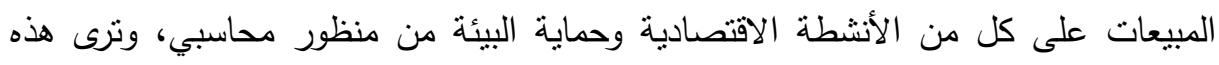

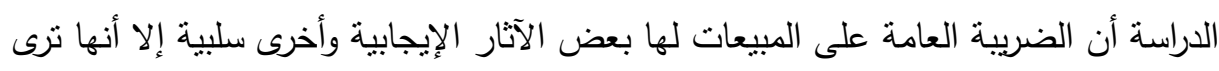

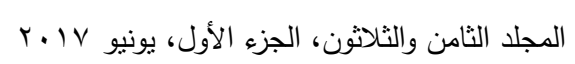


أن الآثار السلبية للضريبة تفوق الآثار الإيجابية، ومن هذه الآثار السلبية: تجميد دوران رأس المال، وزيادة التكاليف وانخفاض الربحية، فقد تبين أن الآثار السلبية للضريبة تفوق الآثار

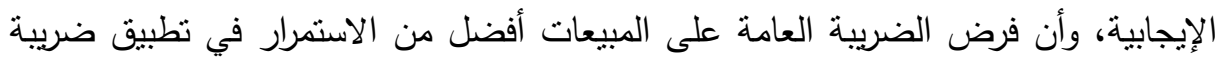
الاستهلاك بكل ما تحمله من عيوب وسلبيات.

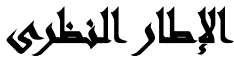

النظام الضريبي: يعد وسيلة فاعلة في يد الدولة خاصة في فترات الركود والأزمات الاقتصادية، إذ يمكن للاولة أن تستعمل النظام الضريبي لتتجيع بعض القطاعات على غيرها من خلال إعفائها من الضرائب لتجاوز مرحلة الركود والأزمات الاقتصادية، مما يترتب عليه لئهيه

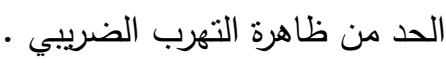
تعريف الضريبة: وعلى ذلك تعرف الضريبة بوجه عام بأنها عبارة عن "|قتطاع نقدي إجباري من الأفراد المساهمة في أعباء الخدات العامة، تبعاً لمقدرتهم على الدفع، ودون النظر إلى المنافع التي تعود عليه من هذه الخدمات، وتستخدم حصيلتها في تحقيق أهداف

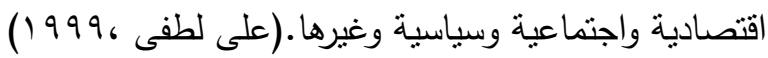
قواعد الضريبة: يقصد بها القواعد التي تحكم فرض الضرائب، ويطلق عليها أيضا قواعد الضريبة تلك الأسس والقواعد التي يتعين على المشرع المالي مراعاتها وهو بصدد تقرير

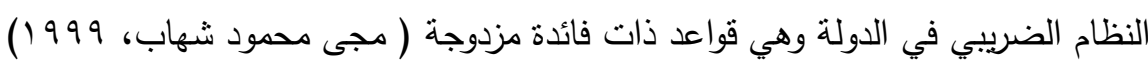

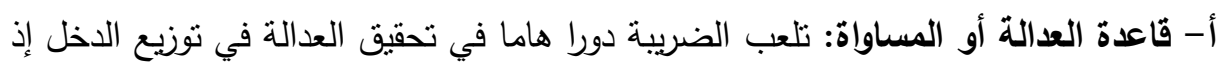
أنها تقتطع نسبة من دخل الفرد، وتختلف باختلاف مصدر الدخل وحجمه وهكذا تميز

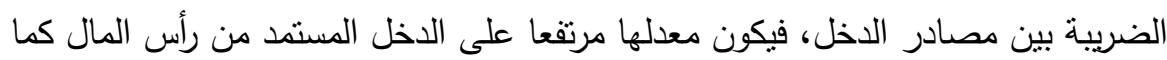

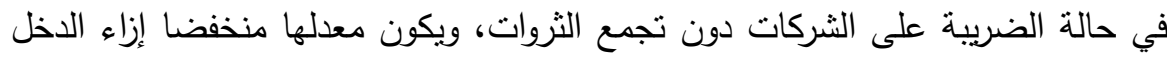

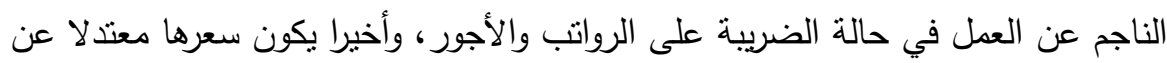

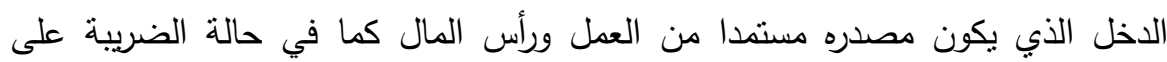


الأرباح التجارية وغير التجارية والصناعبة، وأخيرا تفرض ضريبة إجمالية على عموم

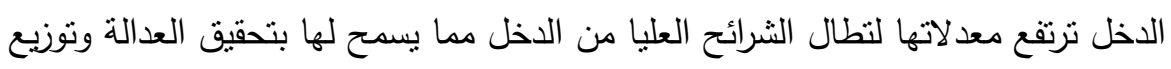

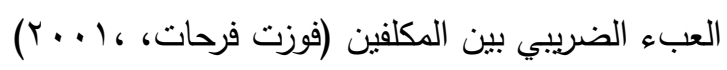

حيث تعتبر العدالة الضريبية من أهم مبادئ النظام الفعال والتي يسعى المشرع الضرين الضريبي

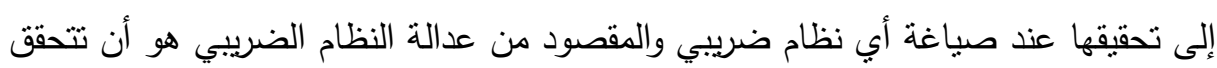

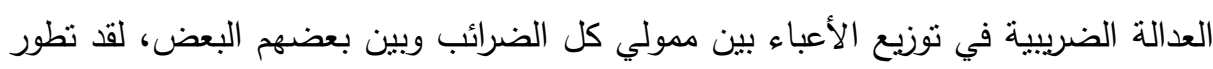

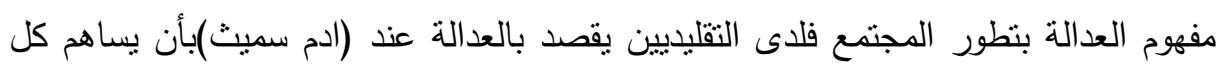
أفراد المجتع في تحمل نفقات الدولة حسب مقدرتهم التكلفية النسبية أي نكون مساهتهم

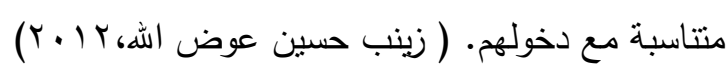

ب. قاعدة اليقين أو التحديد: وتعني هذه القاعدة أن تكون الضريبة محددة ومعلومة وواضحة

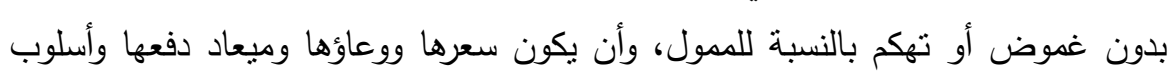
تحصيلها وكل ما يتعلق بأحكامها وإجراءاتها معلوما بصورة مسبقة لاى المكلفين لألفون بأدائها

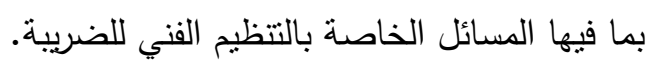

ج- قاعدة الملاعمة في الدفع: تقضي هذه القاعدة أن يكون مبعاد دفع هذه الضريبة مناسبا

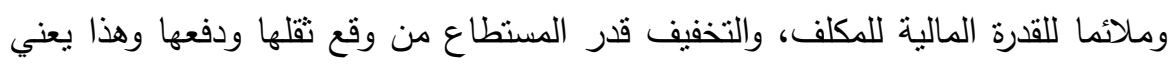

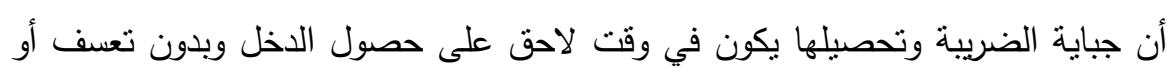

$$
\text { تهكم والتخفيف قدر الإمكان من وطأتها. }
$$

د. قاعدة الاقتصاد في التحصيل: وتعني هذه القاعدة بأن الفارق بين ما يدخل الخزانة العامة

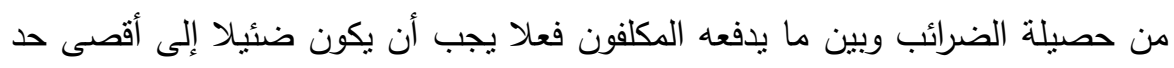

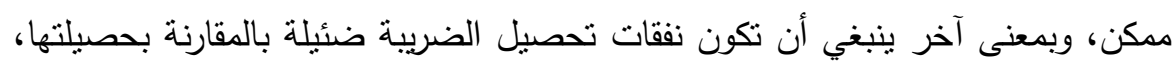

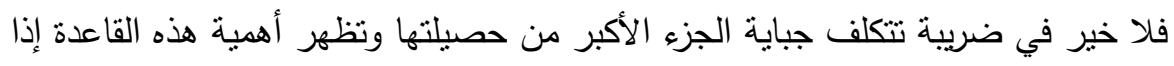

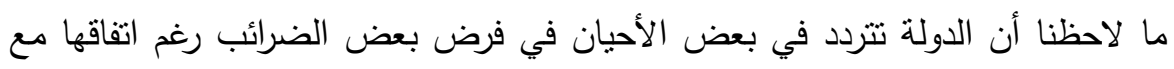

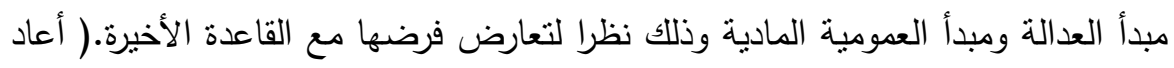

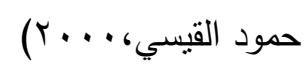


وقد أضاف كتاب المالية الحدثين إضافة للقواعد التي وضعها آدم سميث مايلي: قاعدة الثبات: يقصد بها أن لا تتغير حصيلة الضرائب تبعا للتغيرات التي تطرأ على الحياة الاقتصادية وخصوصا في أوقات الكساد وذلك أن حصيلة الضريبة تزداد عادة في أوقات الرخاء بسبب ازدياد الدخول والإنتاج. قاعدة المرونة: ويقصد بها أن يكون تغير الدخل مصحوبا من الناحية الزمنية وقد ر الأماكن بتغير في حصيلة الضريبة وفي نفس الاتجاه بمعنى أخر الضريبة المرنة هي الني تزداد

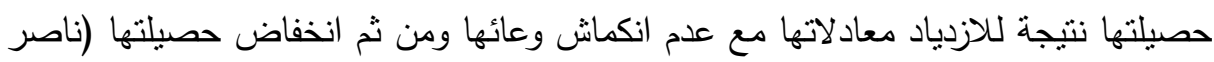

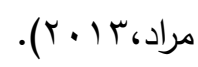

\section{إجراءايه الصراسلا}

1- قام الباحثون بتصميم قائمة إستقصاء تتفق مع أغراض البحث وتساعد على إختبار فروض الدراسة، تكونت عينة الدراسة مكونة من ( (10) مفردة مطبقة على مصلحة الضرائب.

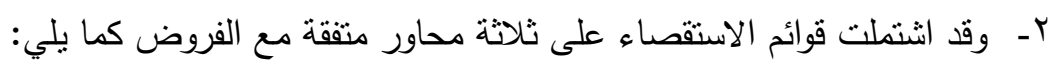

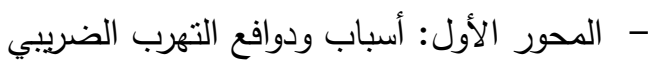

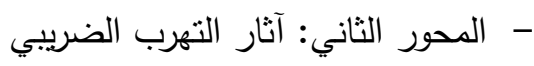
- المحور الثالث: عوامل تساعد على الحد من التهرب الضريبي التئي

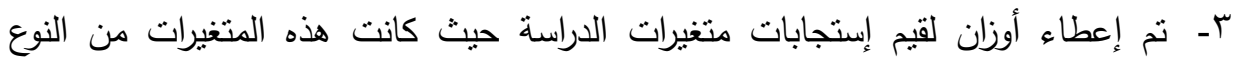
الترتيبى Ordinal Variables وفقاً لمقياس ليكرت الترتيبى الخماسي كما يلى:

\begin{tabular}{|c|c|c|c|c|}
\hline 0 & 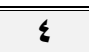 & $\mu$ & $\bar{r}$ & 1 \\
\hline اوافق تماما & اوافق & محايد & ارفض & ارفض بشدة \\
\hline
\end{tabular}


ـ - ت تم إدخال البيانات للحاسب الآلي وقد إستعان الباحث بالبرنامج الإحصائي SPSS، وقامت بالتحليلات النالية:

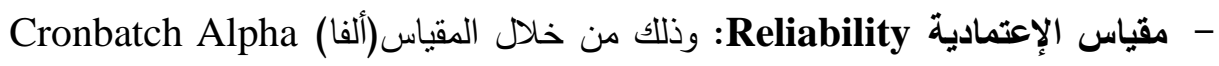

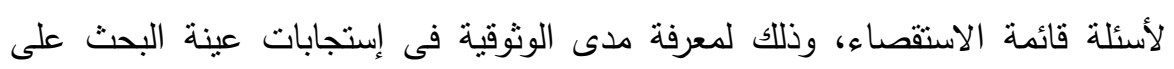

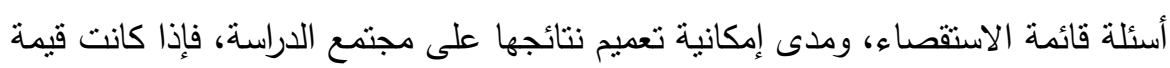

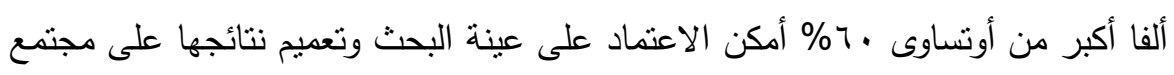
الدراسة.

- التكرارات النسبية: حيث يبين هذا الأسلوب الأوزان النسبية لإستجابات عينة البحث على

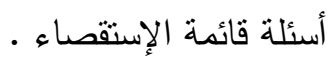

- المتوسط المرجح والإنحراف المعياري: لتوصيف متغيرات الدراسة من حيث النزعة المركزية والتشتت.

وفيما يلى تطبيق هذه الأساليب لإختبار فروض الدراسة: مقاييس الإعتمادية Realiability:

جدول رقم (1) (1)

جدول رقم(1) : مقاييس الإعتمادية Realiability الخاصة بمحاور قائمة الإستقصاء

\begin{tabular}{|c|c|c|c|c|}
\hline معامل الصدق (**) & $\begin{array}{l}\text { معامل الثبات } \\
\text { Cronbatch } \\
\text { alpha }\end{array}$ & عدد العبارت & تعريف & 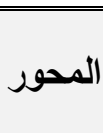 \\
\hline$\% 91,0$ & $\% \wedge \Upsilon, \wedge$ & $\Gamma \varepsilon$ & أسباب ودوافع التهرب الضريبي & الأول \\
\hline$\% \wedge r, r$ & $\% 79, Y$ & YY & آثار التهرب الضريبي & الثانى \\
\hline$\% 91,9$ & $\% \wedge \varepsilon, \varepsilon$ & ro & عوامل تشاعد على الحد من & الثالث \\
\hline
\end{tabular}

(") تم حساب معامل الصدق عن طريق حساب الجذر التربيعي لمعامل الثبات

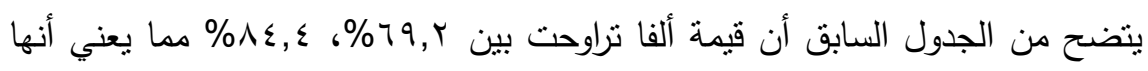

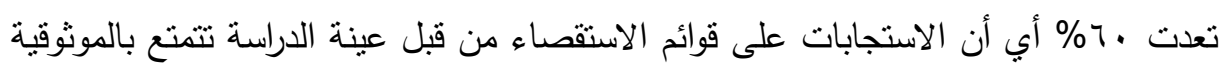
(Realability)، الأمر الذي يمكن معه تعميم نتائج التحليل على مجتمع الدراسة. 


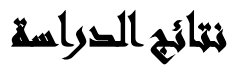

اختبار الفرض الأول: توجد علاقة ذات دلالة معنوية بين التهرب الضريبي وفاعلية النظام الضريبي المصري، وجاءت النتائج كما يلي:

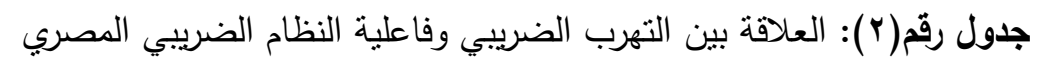

\begin{tabular}{|c|c|c|}
\hline فعالية النظام الضريبي المصري & & \\
\hline$(* *) \cdot, 9 \cdot 9$ & معامل الارنباط & \multirow{2}{*}{ ل لتهرب الضريبي } \\
\hline$\cdot, \cdots 1$ & الدلالة & \\
\hline
\end{tabular}

يتضح من الجدول ما يلي: وجود علاقة ارتباطية ذات دلالة احصائية بين التهرب الضريبي وفاعلية النظام الضريبي المصري حيث كان قيمة معامل الارتباط (9.9 (9. •) بدلالة معنوية

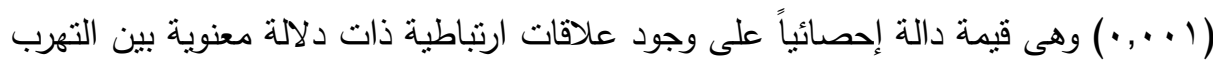
الضريبي وفاعلية النظام الضريبي المصري. اختبار الفرض الثانى: توجد علاقة ذات دلالة معنوية بين التهرب الضريبي والحد من

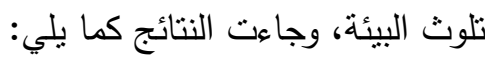
جلول رقم(ץ): العلاقة بين التهرب الضريبي والحد من تلوث البيئة

\begin{tabular}{|c|c|c|}
\hline الآثار البيئية للتهرب الضريبيى & & \\
\hline$(* *) \cdot, 109$ & معامل الازتباط & \multirow{2}{*}{ التهرب الضريبى } \\
\hline$\cdot, \cdots 1$ & الدلالة & \\
\hline
\end{tabular}

يتضح من الجدول ما يلي: وجود علاقة ارتباطية ذات دلالة احصائية بين التهرب الضريبي

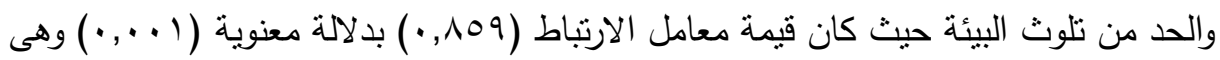
قيمة دالة إحصائياً على وجود علاقات ارتباطية ذات دلالة معنوية بين التهرب الضريبي والحد لهد

$$
\text { من تلوث البيئة. }
$$

اختبار الفرض الثالث: هناك علاقات ارتباط بين التهرب الضريبي والعوامل النشريعية والإدارية والاجتماعية والاقتصادية المسببة للتهرب الضريبي، وجاءت النتائج كما يلي: 
جدول رقم(ع): العلاقة بين التهرب الضريبي والعوامل التتريعية والإدارية والاجتماعية والاقتصادية المسببة للتهرب الضريبي

\begin{tabular}{|c|c|c|}
\hline التهرب الضريبي & & الاسباب \\
\hline$(* *) \cdot$, ro. & معامل الارنباط & \multirow{2}{*}{ أسباب ودوافع تشريعية } \\
\hline$\cdot, \cdot r$ & الدلالة & \\
\hline$(*) \cdot, 170$ & معامل الارتباط & \multirow{2}{*}{ اسباب ودوافع إدارية } \\
\hline$\cdot, \cdot \varepsilon$ & الدلالة & \\
\hline$(* *) \cdot, Y) \leq$ & معامل الارتباط & \multirow{2}{*}{ اسباب ودوافع إجتماعية } \\
\hline$\cdot, \cdots 0$ & الدلالة & \\
\hline$(* *) \cdot, r \vee Y$ & معامل الارتباط & \multirow{2}{*}{ أسباب ودوافع اقتصادية } \\
\hline$\cdot, \cdots 1$ & الدلالة & \\
\hline
\end{tabular}

إثبات صحة الفروض:

- وجود علاقة ارتباطية ذات دلالة احصائية بين التهرب الضريبي واسباب ودوافع تشريعية

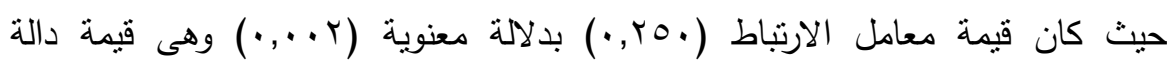

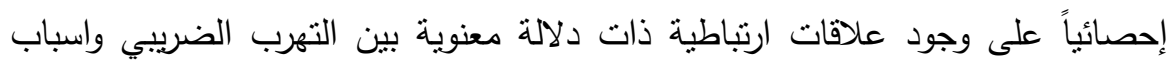
ودوافع تشريعية.

- وجود علاقة ارتباطية ذات دلالة احصائية بين التهرب الضريبي واسباب ودوافع إدارية

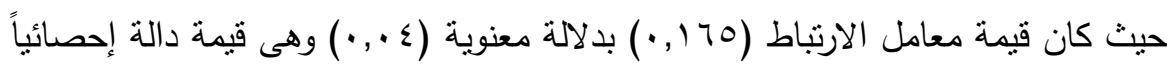
على وجود علاقات ارتباطية ذات دلالة معنوية بين التهرب الضريبي واسباب ودوافع إدارية. - وجود علاقة ارتباطية ذات دلالة احصائية بين التهرب الضريبي واسباب ودوافع إجتماعية

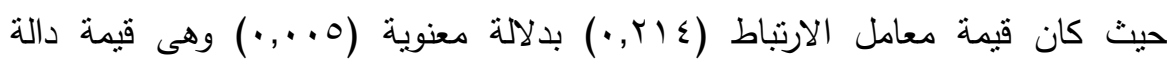

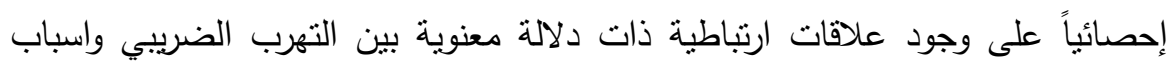
ودوافع إجتماعية. - وجود علاقة ارتباطية ذات دلالة احصائية بين التهرب الضريبي واسباب ودوافع إقتصادية

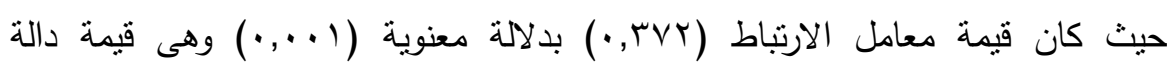

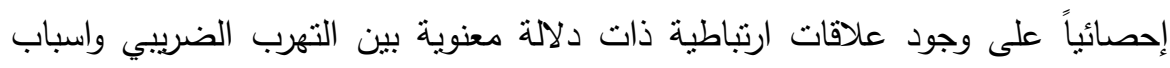
ودوافع إقتصادية. 


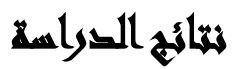

ا ـ تعتبر الضريبة وسيلة فعالة من بين وسائل السياسة المالية للدولة، لما تتمتع به من قدرة على التأثير على الواقع الاقتصادي والاجتماعي، وتتجسد أهمية الضريبة في مختلف لفئل الآثار التي يحدثها التهرب الضريبي على الجانب الاقتصادي والبيئي.

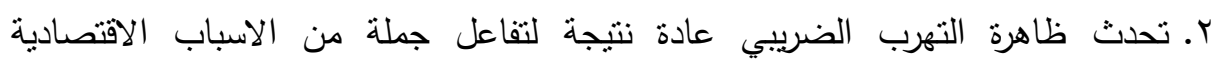
والاجتماعية والادارية، وقد ساعد على استفحالها اتساع ظاهرة العولمة المالية ودورها في انفتاح الاقتصاديات الدولية وتحرير الاسواق العالمية، اضافة الى تطوير وسائل الاتصال.

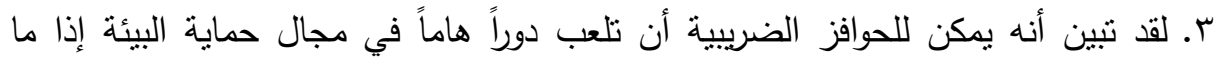
أحسن اختبار أدواتها بما يوفر حافزاً لمصادر التلوث، سواء كانت منشآت أم أفرادا لتخفيض ما يصدر عنها من إحداث تلوث.

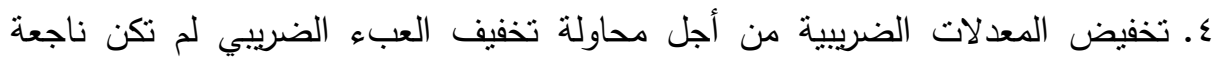

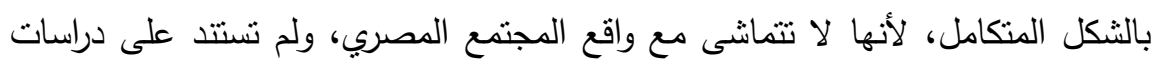

$$
\text { ميدنية مسبقة. }
$$

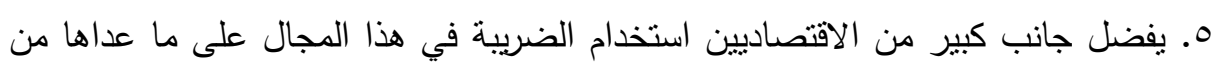

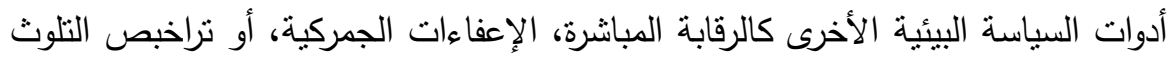
القابلة للاتجار وذلك لقدرتها على تهيئة حافز بصورة مستمرة لمكافحة التلوث، وتدنيتها لتكاليف مكافحة التلوث، وقدرتها على توفير إرادات عالية .

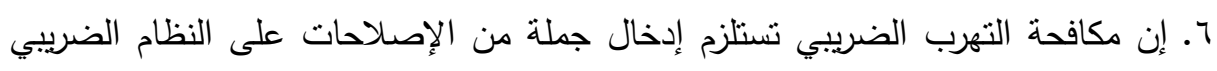
والإدارة الضريبية وتوعية المواطنين وتحفيزهم على الأداء لواجباتهم الضريبية. 


\section{تموسياهي التراسمة}

ا ـ ضرورة نطبيق الضريبة البيئية بجانب عدد من الأدوات الأخرى منل القواعد والمعايير

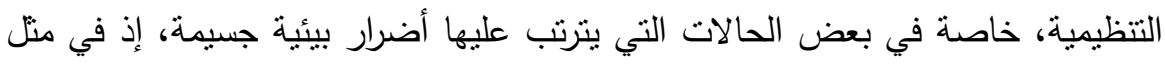
هذه الحالات يكون المنع أو الحظر مقدم على تحصيل الضريبة.

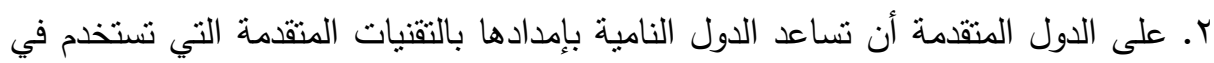
قياس ومكافحة التلوث، فضلا عن تدريب العناصر البشرية التي تعمل في مجال مكافحة

$$
\text { التلوث والحد من ظاهرة التهرب الضريبي. }
$$

r. ضرورة تجميع كافة ما يتعلق بالسياسة الضريبية في مجال مكافحة التلوث سواء ما يتعلق

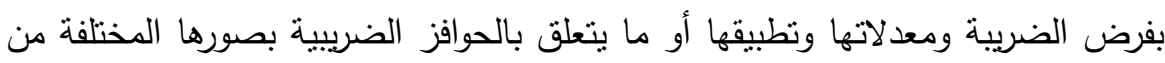

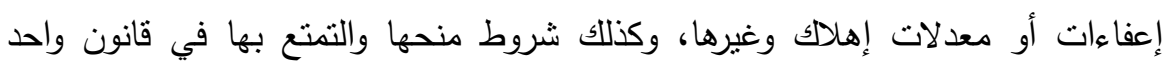
عوضا عن تشتيتها في عدة قوانين مما يساعد على انتشار ظاهرة التهرب الضريبي.

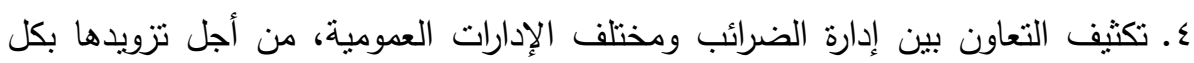
المعلومات والتوضيحات اللازمة حول نشاط المكلفين قصد مكافحة التهرب الضريبي. ه. العمل على نشر الوعي الضريبي لاى المكلف بالضريبة وموظفي الضرائب كذللك، وهذا

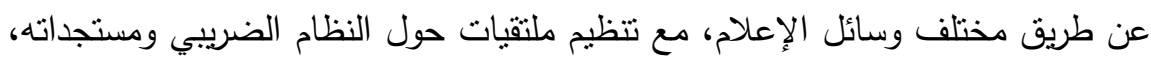
وإدراج الثقافة الضريبية ضمن البرامج التربوية (المدارس، المعاهد والجامعات).

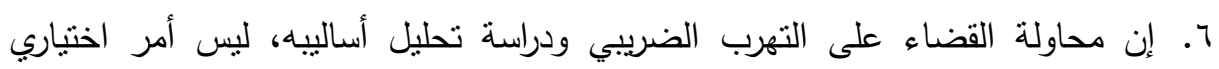
للمشرع والإدارة الضريبية بل هو أمر حتمي، حيث ينبغي تدارك كل النقائص والتتاقضات التي تعتري التشريع الضريبي، وكذلك توفير وتجنيد كل الإمكانيات البشرية والمادية التي بحوزة الإدارة من أجل التحكم والحد من التهرب الضريبي ولوبي نسبيا. V. القضاء على جميع النشاطات التي تغذي ظاهرة التهرب الضريبي، منها الاقتصاد الموازي، والمنافسة غير المشروعة. 
مجلة العلوم البيئية

معهد الدراسات والبحوث البيئية - جامعة عين شمس لئس

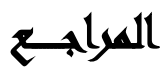

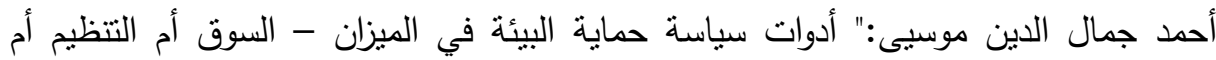

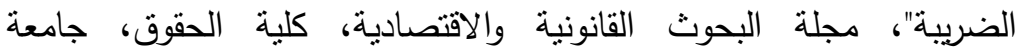

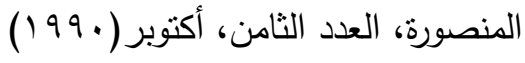

أعاد حمود القيسي( . . ب ):"المالية العامة والتشريع الضريبي"، الطبعة الثالثة، دار النهضة

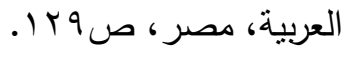

حافظ حسن عوض شعيب(1990): "تأثثر ضرائب المبيعات على كل من الأنشطة

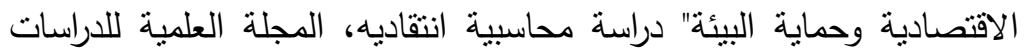

والبحوث التجارية، كلية التجارة ببنها

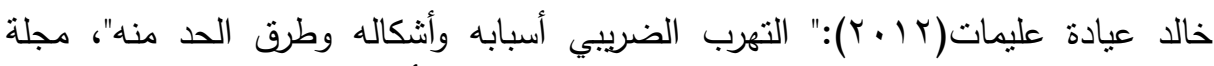

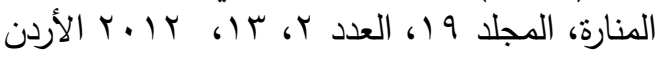

فوزت فرحات( ( . ـ ب):"المالية العامة للاقتصاد المالي"، لبنان، منشورات الحلبي الحقوقية.

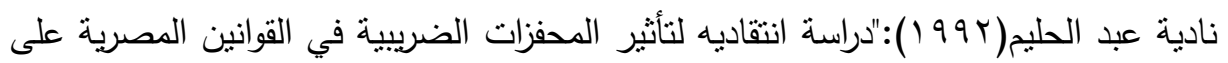

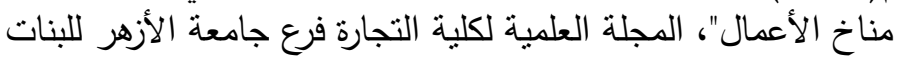

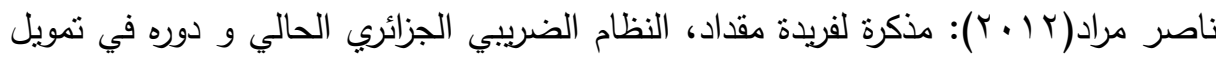
خزينة الدولة، مذكرة تخرج لنيل شهادة لسانس النظان في المالية 


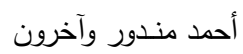

\title{
THE ECONOMIC AND ENVIRONMENTAL IMPACT OF THE PHENOMENON OF TAX EVASION IN EGYPT (FROM 2000 TO 2010)
}

\author{
Mandour, A. ${ }^{(1)}$; AL Saeed, Iman ${ }^{(1)}$ and El-Said, Hoda, F. A. ${ }^{(2)}$ \\ 1) Faculty of Commerce, Ain Shams University 2) Taxation \\ Department
}

\begin{abstract}
The tax evasion, which is the taxpayer's attempt to pay off the payment of his taxes and non-compliance, is a barrier to achieve one of the basic and basic goals in the financing of the treasury revenues sufficient to cover the general needs, at a time when the country suffers from financial difficulties and increase the need to invest in addition to the problems of indebtedness, this has made it necessary to focus on the tax and make it an essential source of treasury financing.

Therefore, any attempt to avoid the negative effects of pollution of the environment necessitates the necessity of pricing and estimating the value of the external effects and introducing these values within the special or internal costs of the economic unit. Partly and nationally, the external cost of pollution is the amount of increased and growing losses to society or the national economy as a result of environmental pollution. Tax evasion is a serious phenomenon that affects the public treasury, where countries miss a significant part of the financial resources. This results in the failure of the state to make public expenditure to the fullest extent. Hence, it is possible to identify the problem and questions of the study. Therefore, the researcher decided to clarify the real dimensions of this phenomenon The positive tax plays on the economy and the preservation of the environment.

Egypt has witnessed a sharp collision in the phenomenon of tax evasion, and if this study is to clarify the importance of the tax as a basic financial resource in any country especially in the Arab Republic of Egypt and the statement of the economic and environmental effects
\end{abstract}

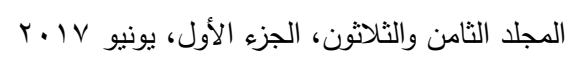


of tax evasion during the period from 2000 to 2010, During this period, and to indicate the impact of this phenomenon on the economy and the environment also to clarify ways and means to combat the phenomenon of tax evasion.

The study included the concept and importance of taxation and its objectives and effects on development, and the importance of taxes in addressing economic and environmental problems through the general budget, to identify the tax evasion and its manifestations in terms of terms and forms and methods of tax evasion, and identify the most important reasons that lead to evasion of payment of tax, As well as the impact of tax evasion on the economy and the environment and explain how to deal with the use of modern trends and how to use tax policies in combating environmental pollution and reduce tax evasion.

The study found that there is a significant statistical correlation between tax evasion and the effectiveness of the Egyptian tax system, as well as the existence of a significant statistical correlation between tax evasion and the reduction of pollution of the environment. The study reached the importance of granting all powers to the competent authority to protect the environment The implementation and implementation of environmental protection, and decentralized administrations to consider granting exemptions and tax incentives, as well as to monitor and verify the implementation of the grant or incentive for exemption . 